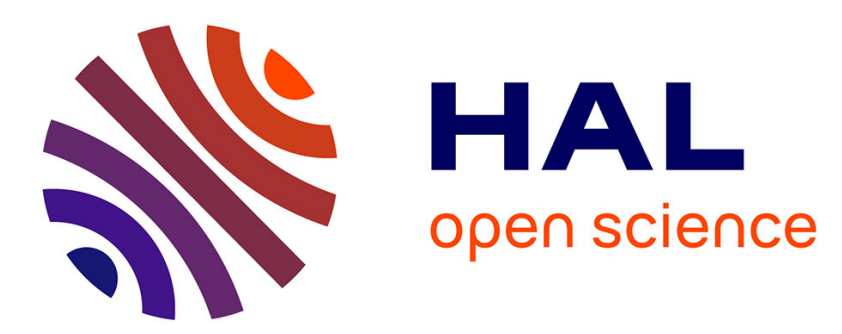

\title{
Dosage de la lignine insoluble en milieu acide : influence du prétraitement par hydrolyse acide sur la lignine Klason de bois et de paille
}

Bernard Monties

\section{- To cite this version:}

Bernard Monties. Dosage de la lignine insoluble en milieu acide: influence du prétraitement par hydrolyse acide sur la lignine Klason de bois et de paille. Agronomie, 1984, 4 (4), pp.387-392. hal00884649

\author{
HAL Id: hal-00884649 \\ https://hal.science/hal-00884649
}

Submitted on 1 Jan 1984

HAL is a multi-disciplinary open access archive for the deposit and dissemination of scientific research documents, whether they are published or not. The documents may come from teaching and research institutions in France or abroad, or from public or private research centers.
L'archive ouverte pluridisciplinaire HAL, est destinée au dépôt et à la diffusion de documents scientifiques de niveau recherche, publiés ou non, émanant des établissements d'enseignement et de recherche français ou étrangers, des laboratoires publics ou privés. 


\section{Dosage de la lignine insoluble en milieu acide : influence du prétraitement par hydrolyse acide sur la lignine Kla- son de bois et de paille}

Bernard MONTIES

I.N.R.A., Laboratoire de Chimie biologique et de Photophysiologie, I.N.A. Paris-Grignon, F 78850 Thiverval-Grignon

RÉSUMÉ

Un protocole de dosage de la lignine insoluble dans l'acide sulfurique concentré, lignine Klason, est décrit : il comporte une étape de préhydrolyse par l'acide sulfurique $5 \%$ et un lavage, en milieu chlorhydrique concentré, du résidu de préhydrolyse. Ce lavage permet d'améliorer la reproductibilité du dosage de la lignine après préhydrolyse et d'atteindre une reproductibilité comparable à celle obtenue par le dosage Klason standard. L'analyse élémentaire des préparations de lignines isolées au cours de ces dosages indique des variations complexes qui dépendent de la nature du produit analysé.

SUMMARY

Determination of acid-insoluble lignin : effect of pretreatment by acid hydrolysis on the Klason lignin in wood and straw.

A procedure for estimating lignin insoluble in concentrated acids, Klason lignin, is described ; it includes a $5 \%$ sulfuric acid prehydrolysis step and washing in concentrated hydrochloric acid. This procedure allowed better reproducibility than without washing, of the same order as that given by the standard Klason procedure. Elemental analysis showed complex changes depending on the plant analysed.

\section{INTRODUCTION}

Lorsque des tissus végétaux lignifiés sont traités par l'acide sulfurique, les polyosides sont hydrolysés et dissous. Le résidu insoluble qui est dosé par gravimétrie, correspond par définition, à la lignine.

Ce type de dosage, simple en apparence, est couramment pratiqué en chimie végétale ; il est pourtant peu satisfaisant. De nombreux auteurs, parfois depuis bien longtemps, ont montré les limites de cette méthode :

- erreurs par excès résultant principalement de la présence de polyosides hémicellulosiques de type xylanes (NORMAN \& JENKINS, 1934a) ou de protéines (NORMAN \& JENKINS, 1934b) ou consécutives aux conditions de séchage des échantillons (MAC DoUGALL \& DELONG, 1948);

- erreurs par défaut attribuées à la fois à la solubilisation d'une fraction des lignines lors des prétraitements qui visent à réduire les contaminations par les hémicelluloses et les protéines : extraction par l'eau bouillante, hydrolyse par l'acide sulfurique dilué à reflux (COHEN \& HARRIS, 1937), et à l'existence de lignine non précipitable après traitement par l'acide sulfurique concentré, dite lignine acido-soluble (CAMPBELl \& MAC DONALD, 1952 ; LAI \& SARKANEN, 1971).

Dans le cas des bois, ce dosage est choisi comme méthode de référence ; la lignine ainsi déterminée est dite : « lignine Klason ». Le protocole Klason est appliqué à la poudre de bois extraite, il consiste en un traitement par l'acide sulfurique concentré à 72 p. 100 suivi, après dilution, d'une posthydrolyse. De nombreuses modifications du dosage Klason ont été proposées; elles ne prennent pas en compte la fraction acido-soluble de la lignine qui n'est pas précipitée après post-hydrolyse (EFFLAND, 1977). Comme les bois contiennent peu de protéines et de xylanes, le protocole Klason ne comporte pas de préhydrolyse par l'acide sulfurique 5 p. 100 sous reflux ; on considère donc comme négligeables les risques d'erreurs par excès liés à la présence de protéines et de xylanes. La lignine Klason est calculée d'après le minimum de masse du résidu insoluble dans l'acide sulfurique après correction de la teneur en cendres. Ce protocole est choisi comme standard alors même qu'il est bien 
établi que le minimum de masse du résidu n'est pas invariablement obtenu avec la concentration standard de 72 p. 100 en $\mathrm{SO}_{4} \mathrm{H}_{2}$ et que la position du minimum dépend des types de bois (FREUDENBERG \& PloETZ, 1940).

Dans le cas des plantes jeunes ou peu lignifiées (pailles et foins, plantes annuelles et bois juvéniles en cours de croissance), le dosage de la lignine insoluble dans les acides pose des problèmes beaucoup plus complexes. Ces végétaux sont en effet relativement plus riches en protéines mais aussi en xylanes et hémicelluloses diverses. De ce fait, des prétraitements hydrolytiques sont systématiquement employés afin de réduire les risques de contamination par les proiéines et les hémicelluloses et cela malgré les possibilités de solubilisation des lignines en milieu acide. Proposée dès 1934 par NORMAN \& JENKINS, la préhydrolyse par l'acide sulfurique est incluse très fréquemment dans les protocoles de fractionnement des parois et de dosage de la lignine (ELLIS et al., 1950 ; JARRIGE, 1961 ; BAILLEY, 1964 ; SOUTHGATE, 1969 ; TOLLIER \& RIQUET, 1980). Ces auteurs ont en particulier admis, de façon parfois implicite, que la teneur en lignine correspond au minimum de masse du résidu après hydrolyses sulfuriques successives. La validité de ce critère quantitatif n'est évidemment pas suffisante; des critères qualitatifs ont donc été proposés pour contrôler la pureté de la lignine de Klason : teneur minimale en azote, teneur maximale en méthoxyles. Ce dernier critère, proposé en 1940 par FREUDENBERG \& PloETZ, est fondé sur le fait que la lignine est formée par polymérisation d'alcools méthoxycinnamiques; il ne prend cependant pas en compte l'hétérogénéité structurale des lignines établie depuis et dont l'importance a été discutée ailleurs (MONTIES \& LAPIERRE, 1981). C'est ainsi que le contrôle des teneurs en méthoxyle ne permet de déterminer avec exactitude ni les variations de teneurs en monomères de type p-hydroxycinnamique (non méthoxylés) présents dans les lignines de Monocotylédones, ni celles des monomères syringyles (diméthoxylés) constituants principaux de la fraction acidosoluble des lignines d'Angiospermes (MUSHA \& GORING, 1974). Paradoxalement, la composition élémentaire en carbone, hydrogène et oxygène pourtant utilisée depuis longtemps pour comparer les préparations de lignine (BRAUNS \& BRAUNS, 1960) n'a pas été systématiquement employée pour comparer les lignines Klason de plantes annuelles obtenues avec ou sans préhydrolyse.

Dans ce travail, nous étudions l'influence de la préhydrolyse sulfurique sur le rendement et sur la composition élémentaire de la lignine Klason de bois et de paille. De plus, des expériences préliminaires ont permis de constater d'importantes différences systématiques entre des séries d'analyses ne différant que par les conditions de lavage des résidus de préhydrolyse ; ces conditions sont délicates à standardiser. Dans ce travail, nous étudions donc aussi l'influence des conditions de lavage des résidus de préhydrolyse sur la reproductibilité des dosages Klason. Pour cela, nous avons modifié un traitement chlorhydrique qui avait été déjà précédemment adapté par MOON \& Abouraya (1952). La lignine obtenue selon ce nouveau protocole est appelée par la suite « lignine avec préhydrolyse et lavage $»$. On a donc comparé la lignine dosée selon ce protocole avec celles obtenues selon un protocole standard «sans préhydrolyse » d'après EFFLAND (1977) et selon un protocole avec " préhydrolyse acide simple " d'après JARRIGE (1961). Dans le cas de la paille de blé, les effets de ces prétraitements sont aussi comparés à ceux d'une préhydrolyse enzymatique qui a pour objet de réduire les risques de contaminations protéiques; le protocole employé est celui de ELLIS et al. (1950). Les compositions élémentaires en carbone, hydrogène, oxygène et azote de chacune de ces préparations ont été aussi analysées.

\section{MATÉRIEL ET MÉTHODES}

Les échantillons (bois de peuplier Populus $x$ euramerica cv. I 214, paille de blé Triticum aestivum cv. "Champlein ») proviennent de plantes récoltées en plein champ ; après séchage à l'air libre et à température ambiante, ils sont réduits en poudre à l'aide d'un micro-broyeur Culatti muni de tamis à trous circulaires de $0,7 \mathrm{~mm}$ de diamètre. La poudre est ensuite traitée au Soxlhet de façon exhaustive successivement à l'aide d'un mélange toluène-éthanol $(2-1, v / v)$ puis d'éthanol puis d'eau. Les résidus pariétaux obtenus sont ensuite séchés par lyophilisation et stockés à $4{ }^{\circ} \mathrm{C}$ à l'abri de l'humidité.

La lignine Klason standard dite «sans préhydrolyse " est estimée suivant le protocole voisin de celui d'EFFLAND (1977). Les prises d'essais de $300 \mathrm{mg}$, pesées à $0,1 \mathrm{mg}$ près, sont soigneusement dispersées dans l'acide sulfurique 72 p. 100 où elles subissent une hydrolyse par l'acide sulfurique à $72 \mathrm{p}$. 100 pendant $2 \mathrm{~h}$ à $20^{\circ} \mathrm{C} \pm 0,5^{\circ} \mathrm{C}$. Après dilution par de l'eau jusqu'à une teneur de 5 p. 100 en acide sulfurique, le mélange obtenu subit une posthydrolyse par reflux sous ébullition douce et agitations périodiques pendant $3 \mathrm{~h}$. Le résidu insoluble, récupéré par centrifugation ou par filtration sur papier en fibre de verre (GFA, WHATMAN) placé sur le verre fritté d'un creuset de GoOCH porosité $n^{\circ} 3$, est ensuite lavé jusqu'à neutralité du filtrat par de l'eau désionisée, 4 fois environ $50 \mathrm{ml}$, puis il est séché à l'étuve jusqu'à poids constant puis incinéré à $550^{\circ} \mathrm{C}$ pendant 3 heures.

La lignine avec «préhydrolyse acide simple » est estimée suivant un protocole voisin de celui de JARRIGE (1961). Les prises d'essais de $300 \mathrm{mg}$ subissent une préhydrolyse par l'acide sulfurique à 5 p. 100 pendant $4 \mathrm{~h}$ sous reflux. Après retour à température ambiante, les résidus d'hydrolyse sont séparés par filtration sur creuset de GOOCH, porosité 1, couvert d'un papier en fibre de verre GFA (WHATMAN) en aspirant lentement sous vide. Le résidu de préhydrolyse resté sur le filtre, lavé 4 fois après remise en suspension par environ $50 \mathrm{ml}$ d'eau désionisée est ensuite séché jusqu'à poids constant à $105^{\circ} \mathrm{C}$. Le résidu $\mathrm{sec}$, appelé lignocellulose, subit le protocole d'analyse standard «sans préhydrolyse » décrit plus haut donnant la lignine « avec simple préhydrolyse ».

La lignine « avec préhydrolyse et lavage » est estimée selon un protocole dérivé du précédent. La préhydrolyse et la filtration du résidu de préhydrolyse sont faites comme précédemment, mais le résidu est 
laissé humide sur filtre. Il est alors immédiatement dispersé par simple agitation dans $25 \mathrm{ml}$ d'acide chlorhydrique fumant. La suspension, laissée à température ambiante, est mise sous agitation lente pendant $15 \mathrm{mn}$ puis elle est diluée par $25 \mathrm{ml}$ d'eau et filtrée. Le résidu de préhydrolyse ainsi traité est lavé successivement par $100 \mathrm{ml}$ d'eau, d'éthanol puis d'éther éthylique, il est enfin séché à $105^{\circ} \mathrm{C}$. Après pesée, la lignocellulose obtenue subit le protocole d'analyse standard sans préhydrolyse décrit plus haut, donnant la lignine "avec préhydrolyse et lavage ". Lors du prétraitement par hydrolyse enzymatique selon ELLIS et al., (1950), l'hydrolyse pepsique est faite à $37^{\circ} \mathrm{C}$ pendant $24 \mathrm{~h}$ dans l'acide chlorhydrique $0,1 \mathrm{~N}$ sous agitation douce ; après lavage sur filtre, la teneur en lignine đu résidu de cette préhydrolyse est déterminée par dosage avec préhydrolyse simple.

La composition élémentaire en azote, carbone, hydrogène et oxygène dosés par méthode électrochimique, est calculée d'après les analyses faites par le laboratoire de micro-analyses du CNRS (69390 Vernaison, France). Les résultats, corrigés de la teneur en cendres, sont la moyenne de 3 déterminations indépendantes.

Les réactifs du commerce, les plus purs (PROLABO, RP, MERCK, PA) sont utilisés directement. L'acide chlorhydrique fumant est à 37 p. 100 , densité environ 1,19 .

\section{RÉSULTATS}

Les tableaux 1 et 2 donnent les variations du rendement et de la composition centésimale des 3 types de lignines obtenues à partir de bois de peuplier.

Dans le tableau 1 les teneurs en lignocellulose ont été utilisées pour calculer, par différences, les teneurs en polyosides aisément solubilisés en milieu acide (PAS) et en polyosides difficilement solubilisés en

\section{TABLEAU 1}

Composition chimique comparée du bois juvénile de peuplier en fonction du mode de prétraitement appliqué avant dosage de la lignine insoluble en milieu acide. Moyenne \pm écart type en p. 100 de la masse du résidu pariétal $(R P)$. Les teneurs en matériel pariétal aisément soluble en milieu acide $\left(P_{A S}\right)$ et en matériel difficilement solubles en milieu acide (PDS) ont été calculées par différence entre la lignocellulose $(L C)$, la lignine $(L)$, les cendres $(D)$ selon les relations $P_{A S}=R P-L C$ et $P D S=L C-L-D$.

Comparative chemical composition of juvenile poplar wood according to pretreatment before analysis of acid-insoluble lignin. Mean \pm standard deviation as $\%$ of residual wall material. The contents of readily acid-soluble material $\left(P_{A S}\right)$ and poorly acid-soluble material (PDS) were calculated by difference between lignocellulose $(L C)$, lignin (L) and ash (D) by the equations $P A S=R P-L C$ and

$$
P D S=L C-L-D \text {. }
$$

\begin{tabular}{lcccc}
\hline \hline $\begin{array}{c}\text { Prétraitement } \\
\text { par } \\
\text { préhydrolyse }\end{array}$ & $\begin{array}{c}\text { Lignine } \\
\text { (L) }\end{array}$ & $\begin{array}{c}\text { Ligno- } \\
\text { cellulose } \\
\text { (LC) }\end{array}$ & PDS & PAS \\
\hline sans & $23,3 \pm 0,7$ & n.d () & n.d & n.d \\
\hline simple & $19,5 \pm 2,4$ & $60,5 \pm 1,9$ & $40,3 \pm 0,8$ & $39,5 \pm 1,9$ \\
\hline avec lavage & $16,9 \pm 0,4$ & $54,9 \pm 1,0$ & $37,4 \pm 0,5$ & $45,1 \pm 1,0$ \\
\hline \hline
\end{tabular}

() n.d = non déterminé. milieu acide (PDS) après la préhydrolyse. Alors qu'il est bien établi que la préhydrolyse a pour effet de solubiliser les hémicelluloses qui constituent la majeure partie des PAS, l'expression «polyosides aisément solubles " a été retenue car il n'est pas possible d'exclure la présence de fragments de cellulose et de lignine dans cette fraction. La même remarque est valable pour les PDS constitués essentiellement de cellulose. Les moyennes et écart-type indiqués dans le tableau 1 correspondent à 5 séries de 6 analyses parallèles comportant 3 répétitions de 2 des dosages comparés; les hydrolyses de la lignocellulose par l'acide sulfurique 72 p. 100 puis les posthydrolyses ont été faites simultanément dans chacune des séries. Dans chaque série, le dosage avec préhydrolyse simple a été pris comme référence; les résultats du tableau 1 correspondent donc aux moyennes de 10 séries de dosages avec préhydrolyse simple et de 5 séries pour les 2 autres types de dosage.

On constate que les reproductibilités du dosage sans préhydrolyse et du dosage avec préhydrolyse et lavage sont supérieures à celles du dosage avec préhydrolyse simple. De plus, les teneurs en lignine insoluble en milieu acide diffèrent significativement; elles décroissent dans l'ordre : lignine sans préhydrolyse, lignine avec préhydrolyse simple et lignine avec préhydrolyse et lavage. Le lavage chlorhydrique du résidu de préhydrolyse provoque aussi une diminution significative du rendement en lignocellulose à laquelle correspond une augmentation de la quantité des polyosides aisément solubilisés. Les compositions centésimales indiquées dans le tableau 2 sont arrondies à la première décimale qui est exacte à $\pm 0,1$ près. On constate que, dans tous les cas, les prétraitements affectent significativement la teneur en carbone qui augmente et la teneur en oxygène qui diminue. Par contre, les teneurs en hydrogène et en azote ne varient pas significativement.

\section{TABLEAU 2}

Composition centésimale comparée des préparations de lignine de peuplier en fonction du mode de prétraitement appliqué avant dosage de la lignine insoluble en milieu acide. Moyennes de 3 répétitions.

$\%$ elemental composition of preparations of poplar lignin according to pretreatment before analysis. Means of 3 replicates.

\begin{tabular}{lcccc}
\hline $\begin{array}{c}\text { Prétraitement } \\
\text { par } \\
\text { préhydrolyse }\end{array}$ & $\mathrm{C}$ & $\mathrm{H}$ & $\mathrm{O}$ & $\mathrm{N}$ \\
\hline sans & 57,7 & 5,7 & 37,9 & 0,2 \\
\hline simple & 63,4 & 5,8 & 32,1 & 0,2 \\
\hline avec lavage & 61,1 & 5,5 & 32,2 & 0,2 \\
\hline \hline
\end{tabular}

Le tableau 3 présente le bilan des effets des prétraitements dans le cas de la paille de blé.

Comme dans le cas du peuplier, on constate que la préhydrolyse et surtout le lavage réduisent de façon significative les teneurs en lignine et en lignocellulose. On constate encore que le protocole avec préhydrolyse simple est moins reproductible que les protocoles sans préhydrolyse et avec préhydrolyse et lavage. 


\section{TABLEAU 3}

Composition chimique comparée de la paille de blé en fonction du mode de prétraitement appliqué avant dosage de la lignine en milieu acide. Moyenne \pm écart type en p. lo0 de la masse de résidu pariétal pour 2 séries de 3 répétitions de chacun des dosages à l'exception du dosage avec préhydrolyse pepsique répété seulement 3 fois. Abréviations et calculs selon tableau 1.

Comparative chemical composition of wheat straw according to pretreatment before analysis. Mean \pm standard deviation as $\%$ of residual wall material for 2 sets of 3 replicates for all determinations except for pepsic prehydrolysis ( 3 replicates only). Otherwise as for table I.

\begin{tabular}{lcccc}
\hline \hline $\begin{array}{c}\text { Prétraitement } \\
\text { par } \\
\text { préhydrolyse }\end{array}$ & $\begin{array}{c}\text { Lignine } \\
(\mathrm{L})\end{array}$ & $\begin{array}{c}\text { Ligno- } \\
\text { cellulose } \\
\text { (LC) }\end{array}$ & PDS & PAS \\
\hline sans & $18,2 \pm 0,1$ & n.d () & n.d & n.d \\
\hline simple & $14,6 \pm 0,8$ & $62,4 \pm 0,5$ & $47,0 \pm 0,5$ & $37,6 \pm 0,5$ \\
\hline $\begin{array}{l}\text { simple avec } \\
\text { hydrolyse } \\
\text { pepsique }\end{array}$ & $12,0 \pm 0,9$ & $56,5 \pm 1,1$ & $43,7 \pm 0,6$ & $43,5 \pm 1,1$ \\
\hline avec lavage & $12,9 \pm 0,4$ & $54,1 \pm 0,8$ & $40,5 \pm 0,9$ & $45,9 \pm 0,8$ \\
\hline \hline
\end{tabular}

(†) n.d = non déterminé.

Le dosage avec préhydrolyses pepsique et acide ne réduit pas de façon significative la teneur en lignine par rapport au protocole avec lavage; de plus, il entraîne une variation peu significative de la teneur en lignocellulose. Les teneurs en cendres de ces préparations n'étant pas significativement différentes, les variations des teneurs en PDS et PAS sont-elles aussi peu différentes.

Dans le tableau 4, on constate que le traitement pepsique ne réduit pas plus significativement la teneur en azote de la lignine que ne le font les autres prétraitements. A l'exception de la préhydrolyse enzymatique, les prétraitements entraînent une réduction de la teneur en oxygène déjà observée dans le cas du peuplier. Par contre, la teneur en carbone des lignines de blé diminue systématiquement après chacun des prétraitements.

\section{TABLEAU 4}

Composition centésimale comparée des préparations de lignine de blé en fonction du mode de prétraitement appliqué avant dosage de la lignine insoluble en milieu acide.

$\%$ elemental composition of wheat lignin preparations according to pretreatment before analysis.

\begin{tabular}{lcccc}
\hline $\begin{array}{l}\text { Prétraitement } \\
\text { par } \\
\text { préhydrolyse }\end{array}$ & $\mathrm{C}$ & $\mathrm{H}$ & $\mathrm{O}$ & $\mathrm{N}$ \\
\hline sans & 53,6 & 5,2 & 31,4 & 1,1 \\
\hline simple & 52,2 & 5,0 & 29,1 & 0,5 \\
\hline $\begin{array}{l}\text { simple avec } \\
\text { hydrolyse } \\
\text { pepsique }\end{array}$ & 52,6 & 5,3 & 31,3 & 0,6 \\
\begin{tabular}{l} 
avec lavage \\
\hline
\end{tabular} & 51,5 & 5,2 & 29,3 & 0,5 \\
\hline
\end{tabular}

\section{DISCUSSION ET CONCLUSIONS}

Quel que soit le matériel analysé, on constate que le lavage chlorhydrique pratiqué après la préhydrolyse par l'acide sulfurique 5 p. 100 provoque, d'une part, une augmentation de la reproductibilité du dosage de la lignine et, d'autre part, une chute des rendements en lignine et en lignocellulose. Par contre, ce même traitement ne modifie pas de la même façon la composition élémentaire des lignines correspondantes.

Alors que le lavage chlorhydrique augmente significativement la reproductibilité du dosage de la " lignine avec préhydrolyse » d'une série d'analyse à une autre, il convient de souligner que, à l'intérieur d'une même série de répétitions, la reproductibilité des 3 types de dosage est voisine. Cette reproductibilité mesurée par le pourcentage de l'écart-type à la moyenne est pour les 3 dosages de l'ordre de 4 p. 100. La faible reproductibilité observée dans le tableau 1 pour les dosages par simple hydrolyse résulte, le plus souvent, de variations systématiques de la moyenne d'une série d'analyses à une autre. Le cas du bois de peuplier pour lequel le nombre de séries est élevé permet de discuter ces faits en détail. Sur 10 séries de 3 répétitions du dosage de la lignine avec préhydrolyse simple, la dispersion moyenne des résultats, mesurée par l'écart type, varie entre 0,1 et 0,9 avec une valeur moyenne de 0,4 alors que les teneurs moyennes correspondantes varient entre 15,8 et 23,0 . Ces 2 valeurs extrêmes des séries de dosage de lignine avec préhydrolyse simple ne sont pas significativement différentes des teneurs en lignine trouvées respectivement par le dosage avec préhydrolyse et lavage et par le dosage sans préhydrolyse (tabl. 1). On peut donc conclure que le dosage chlorhydrique permet d'éliminer de façon reproductible des produits qui sont systématiquement comptés dans la lignine Klason « standard 》 et qui figurent de façon aléatoire dans la lignine Klason " avec préhydrolyse simple ».

Dans le cas des pailles, l'amélioration de reproductibilité consécutive au lavage est moins nette ; le nombre plus réduit de séries d'analyses pratiquées semble suffisant pour expliquer cette observation.

Le lavage chlorhydrique a donc pour effet d'éliminer, de façon reproductible, du matériel pariétal rendu solubilisable par préhydrolyse. Il en résulte, dans tous les cas, une chute du rendement en lignocellulose qui peut résulter principalement de la solubilisation de lignine et d'hémicellulose mais aussi, à un degré moindre, de protéines dans le cas du blé.

La comparaison des variations de composition élémentaire des différentes préparations de lignine permet de préciser ces points. La préhydrolyse simple suffit pour réduire, de près de $50 \mathrm{p}$. 100, la teneur en azote des préparations de lignine de paille. L'effet n'est pas net sur peuplier ; ce résultat n'est pas inattendu dans la mesure où les bois sont pauvres en matières azotées. Par contre, et même dans le cas de bois, les préparations de lignines ne sont jamais pures de contaminations azotées; le lavage chlorhydrique ne réduit pas ce type de contamination. Il ne faudrait cependant pas en déduire que ce lavage ne modifie pas les compositions élémentaires. En effet, dans le cas de la lignine de bois, on constate un effet spécifique du lavage sur la teneur en carbone. Alors que la 
simple préhydrolyse réduit uniformément les teneurs en oxygène des lignines et provoque une augmentation de teneur en carbone, la lignine obtenue après préhydrolyse et lavage est relativement moins riche en carbone que son homologue obtenue par préhydrolyse simple.

Puisqu'il est bien établi que la préhydrolyse a pour effet de solubiliser des hémicelluloses qui constituent la majeure partie du matériel pariétal aisément soluble et puisque le lavage chlorhydrique modifie l'effet de la préhydrolyse, on peut supposer que la variation de composition élémentaire observée après ces 2 types de prétraitements résulte de l'élimination d'hémicelluloses. Les hémicelluloses sont en effet relativement plus riches en oxygène et plus pauvres en carbone que les lignines. L'élimination des hémicelluloses, lors de prétraitement, augmenterait donc le caractère aromatique des préparations de lignine qui sont effectivement plus riches en carbone et plus pauvres en oxygène après prétraitement. Cette hypothèse concorde bien avec les conclusions de NORMAN \& JENKINS, non infirmées depuis 1934, qui ont montré que les hémicelluloses de paille sont susceptibles de former des produits insolubles et non hydrolysables qui sont identifiés, par gravimétrie, à la lignine Klason. Comme cependant les variations de composition élémentaires des préparations de lignine de blé ne concordent que partiellement avec les variations observées dans le cas de peuplier, on peut conclure que l'élimination des hémicelluloses par préhydrolyse et lavage ne permet pas d'expliquer systématiquement les effets qualitatifs et donc quantitatifs des prétraitements lors des différents dosages de lignine. Il est vraisemblable que la diversité des hémicelluloses (JOSELEAU, 1980) et l'hétérogénéité des lignines (MONTIES, 1980) sont en partie responsables de ces différences. Faute de savoir caractériser finement les lignines précipitées en milieu sulfurique, il est très difficile de rechercher dans ces dernières des indices de présence de produits dérivés d'hémicelluloses. Dans le cas de ces lignines, on pourrait envisager par contre de contrôler, suivant FreUdenberG \& Ploetz (1940), l'influence des prétraitements sur les teneurs en méthoxyle des préparations de lignine. Cependant, l'interprétation des résultats de ce type de contrôle est rendue hasardeuse par la possibilité de perte sélective de fragments de lignine relativement enrichis en motif syringyle sous forme de lignine acido-soluble. La solubilisation sélective de motif syringyle est, en effet, suffisante pour réduire la teneur en méthoxyle des préparations de lignine sans que cependant la pureté de la fraction de lignine résiduelle soit modifiée. Il est tout à fait vraisemblable, à ce propos, que les discordances, restées inexpliquées, entre minimum de masse des lignines insolubles dans l'acide sulfurique concentré et maximum de teneur en méthoxyle, rapportées pour certains bois par FreudenberG \& Ploetz dès 1940, résultent précisément de ce type de perte spécifique. C'est pour cette raison que l'étude globale des teneurs en méthoxyles des préparations de lignine n'est pas faite dans ce travail.

Par contre, l'étude des produits de dégradation des lignines solubilisées après préhydrolyse et lavage chlorhydrique devrait permettre de mieux comprendre non seulement l'effet des prétraitements mais encore la nature de la lignine acido-soluble. Au cours d'expériences de marquage de lignine de bois de peuplier et de tiges de blé à l'aide de phénylalanine ${ }^{14} \mathrm{C}$, nous avons constaté invariablement la présence de fortes radioactivités dans les fractions de résidus pariétaux solubilisées par hydrolyses sulfuriques (BAYET \& MONTIES, 1979, résultats non publiés; BAYET, 1980 ; ODIER et al., 1981 ; AGOSSIN et al., 1982). Ces fractions pourraient être constituées, au moins en partie, par des fragments monomériques du type « cétone de Hibbert ». La formation de substances de ce type a été observée par ITO et al. (1981) lors d'hydrolyses sulfuriques de molécules modèles de lignine ayant la structure arylglycérol- $\beta$-aryl éther. Des études sont en cours pour préciser la nature et l'éventuelle signification biosynthétique de la fraction de lignine solubilisée par préhydrolyse qui pourrait correspondre à des fractions tardivement incorporées au polymère et donc relativement peu condensées.

Quelle que soit cette signification, les résultats obtenus démontrent que les dosages réalisés selon le protocole standard «sans préhydrolyse » et selon le protocole de dosage "avec préhydrolyse et lavage» fournissent, avec une bonne reproductibilité, des valeurs vraisemblablement par excès pour le premier dosage, et certainement par défaut pour le second, de la teneur en lignine. Ils sont cependant tous deux préférables au dosage " avec préhydrolyse simple » qui est moins reproductible.

De plus, les variations de composition élémentaire observées après différents prétraitements permettent de conclure que les différentes préparations de lignine isolées selon différents protocoles et à partir de différentes plantes ne sont pas strictement homologues.

La caractérisation physico-chimique de la fraction de lignine facilement solubilisée par préhydrolyse devrait permettre de préciser l'intérêt respectif du dosage avec préhydrolyse et lavage et du dosage standard sans préhydrolyse.

Recu le 9 novembre 1983. Accepté le 23 décembre 1983.

\section{REMERCIEMENTS}

Dominique Jouin et Monique Gaudillère ont contribué à ce travail avec un dynamisme et une compétence technique que l'auteur tient à mentionner avec gratitude.

\section{RÉFÉRENCES BIBLIOGRAPHIQUES}

Agossin E., Odier E., Gaudillère M., Monties B., 1982. Biodégradabilité de lignines ${ }^{14} \mathrm{C}$ de blé par Sporotrichum pulverulentum Nov. en fonction de leur position dans le dernier entrenœud de la tige. Bull. Groupe Polyphénols (C.R. J.E.P. 83, Toulouse), 11, 187-195.
Bailey R. W., 1964. Pasture quality and ruminant nutrition. I. Carbohydrate composition of ryegrass varieties grown as sheep pastures. N.Z. J. Agric. Res., 7, 496-507.

Bayet F., 1980. Etude de la lignine et des acides phénoliques de la tige de blé. Thèse Docteur-Ingénieur, INA-PG, 68 p. I.N.R.A. edit. 
Brauns F. E., Brauns D. A., 1960. The elementary composition and constituent groups in lignin. In : "The Chemistry of Lignin », Supplement volume Acad. Press, Pub., New York, 227 p.

Campbell W. G., Mc Donald I. R. C., 1952. The chemistry of wood cell wall. II. - Isolation of Beech and Spruce acide soluble and modified lignins. J. Chem. Soc., 3180-3183.

Cohen W. E., Harris E. E., 1937. Pretreatment of wood with hot dilute acid : effect on lignin values. Ind. Eng. Chem. Anal., 9, 234235 .

Effland M. J., 1977. Modified procedure to determine acidinsoluble lignin in wood and pulp. TAPPI, 60, 143-144.

Ellis G. H., Matrone G., Maynard L. A., 1950. A 72 percent $\mathrm{H}_{2} \mathrm{SO}_{4}$ method for the determination of lignin and its use in animal nutrition studies. J. Anim. Science, 5, 285-287.

Freudenberg K., Ploetz T., 1940. Die quantitative Bestimmung des Lignins. Chem. Berichte, 73, 754-757.

Ito T., Terashima N., Yasuda S., 1981. Chemical structure of sulfuric acid lignin. III. - Reaction of arylglycerol- $\beta$-aryl ether with five percent sulfuric acid. Mokuzai Gak, 27, 484-490.

Jarrige R., 1961. Analyse des constituants glucidiques des plantes fourragères. I. - Fractionnement des constituants par les hydrolyses acides. Ann. Biol. Anim. Biochem. Biophys., 1, 163-212.

Joseleau J. P., 1980. Les hémicelluloses. In « Les polymères végétaux: polymères pariétaux et alimentaires non azotés, p. 87-121. Monties B., edit. Gauthier-Villars pub. Paris, 345 p.

Lai A. Z., Sarkanen K. V., 1971. Isolation and structural studies, p. 190-195, Chap. 5. In «Lignins », Sarkanen K. V. \& Ludwig C. H. eds, Wiley pub., $916 \mathrm{p}$.
Mac Dougall D., Delong W. A., 1948. A method for the determination of the lignin content of fresh plant tissue without preliminary drying. Can. J. Res., 26B, 468-471.

Monties B., 1980. Les lignines. In «Les Polymères végétaux: polymères pariétaux et alimentaires non azotés », p. 122-155. Monties B., ed. Gauthier-Villars pub. Paris, 345 p.

Monties B., Lapierre C., 1981. Hétérogénéité des lignines. Physiol., Vég., 19, 327-348.

Moon F. E., Abou-Raya A. K., 1952. The lignin fraction of animal feeding-stuffs. II. - Investigation of the lignin determination procedure and development of a method for acid-insoluble lignin. J. Sci. Food Agric., 3, 407-418.

Musha Y., Goring D. A. I., 1974. Klason and acid-soluble lignin content of hardwoods. Wood Sci., 7, 133-134.

Norman A. G., Jenkins S. H., 1934a. The determination of lignin. I. - Errors introduced by the presence of certain carbohydrate. Biochem. J., 28, 2147-2159.

Norman A. G., Jenkins S. H., 1934b. The determination of lignin. II. - Errors introduced by the presence of proteins. Biochem. J., 28, 2160-2168.

Odier E., Janin G., Monties B., 1981. Poplar lignin decomposition by gram-negative aerobic bacteria. Appl. Env. Microbiol., 41, 337-341.

Southgate D. A. T., 1969. Determination of carbohydrate in foods. II. - Unavalaible carbohydrate. J. Sci. Food Agric., 20, 331-336.

Tollier M. T., Riquet A. M., 1980. Dosage des polyosides. In : "Les polymères végétaux : polymères pariétaux et alimentaires non azotés ». 345 p. Monties B., ed., Gauthier-Villars pub., Paris, 157175. 